\title{
Metabolite profiling and assessment of metabolome compartmentation of soybean leaves using non-aqueous fractionation and GC-MS analysis
}

\author{
Noureddine Benkeblia · Takuro Shinano • \\ Mitsuru Osaki
}

Received: 8 February 2007/ Accepted: 3 July 2007 / Published online: 31 August 2007

(C) Springer Science+Business Media, LLC 2007

\begin{abstract}
In the present study, non-aqueous fractionation (NAQF) and GC-MS were used to obtain a spatially resolved view of metabolism in mature leaves of soybean (Glycine max Merr.). NAQF of lyophilized soybean leaves was performed using $\mathrm{CCl}_{4}-n$-heptane and ultracentrifugation that yielded a gradient comprised of six fractions. Chlorophyll content, and marker enzyme activities, phosphoenolpyruvate carboxylase (PEPC) and $\alpha$-mannosidase, were utilized as stroma, cytosol and vacuole markers, respectively. GC-MS analyses of each fraction resulted in the identification of around 100 different metabolites. The distribution of these identified compounds showed a decreasing order from the vacuole to cytosol to chloroplast stroma. In other words, a greater number of identified compounds were found in the vacuole when compared to the cytosol or stroma.

Levels of sugars, organic acids and fatty acids showed greater relative abundances in the vacuole with 50, 55, and $50 \%$ of the respective pools. A greater relative abundance of amino acids was observed in the cytosol where $45 \%$ of the total of amino acids content was recorded. The relatively large pool of sugars and phenolic acids in the vacuole compartment implies high levels of starch metabolism and phenylpropanoid biosynthesis. The low amino acids pool, on the other hand, suggests low nitrogen accumulation in the leaves of soybean. Hierarchical cluster analysis on the most abundant metabolites revealed three clusters containing 10, 20, and 2 of the 32 selected metabolites. The
\end{abstract}

N. Benkeblia $\cdot$ M. Osaki

Graduate School of Agriculture, Hokkaido University,

Sapporo 060-8589, Japan

T. Shinano $(\varangle)$

CRIS, Hokkaido University, Sapporo 001-0021, Japan

e-mail: takuro@chem.agr.hokudai.ac.jp data were discussed in term of NAQF and GC-MS analysis of soybean mature leaves, and also in term of distribution and compartmentation of metabolites at subcellular levels.

Keywords Compartmentation - GC-MS · Metabolome · Non-aqueous fractionation - Metabolite Profiling .

Soybean (Glycine max Merr.)

\section{Introduction}

A principal goal of metabolomics is the measurement of the complete range of primary and secondary metabolites present within, or transported from, a biological system as a means to better understand the precise biological role or function of that system at a defined developmental stage or under specific environmental factors. The search for new trait-enhanced crops, of course, renders it necessary to continue to develop new insights into the response of plants to genetic and environmental changes induced by biotic or abiotic stresses. Metabolite profiling and metabolic pathways mapping can be considered as critical steps (Fiehn et al. 2000; Kell 2004) in providing such information.

A metabolomics experiment typically extends from sampling and sample preparation through analytical strategies for data acquisition to statistical and bioinformatics approaches to raw data processing and data analysis (Brown et al. 2005). Given the diverse physico-chemical nature of different metabolites, no single analytical technology can suffice to cover the entire metabolome. However, the range of current comprehensive technologies, including chromatographic techniques coupled to massspectrometry, currently facilitates extensive coverage of a wide array of metabolites associated with important 
biosynthetic pathways and processes (Dunn and Ellis 2005). Yet a somewhat underrepresented approach to metabolomic studies relates to assessments of the relative distribution of metabolites in different plant compartments. Whilst clearly a technical challenge, approaches to subcellular fractionation that minimize biochemical degradation during sample preparation are known. In principle, these can be combined with current developments in metabolic profiling to more accurately reflect the distribution of metabolic processes within a cell or tissue. We have initiated preliminary evaluations of this approach when applied to soybean leaf and this is the focus of this paper.

Compartmentalization of metabolites and metabolic process is an important aspect of metabolic regulation; metabolite compartmentalization constitutes a principal regulatory characteristic of plant metabolism (Fridman and Pichersky 2005; Goodacre et al. 2004; Lunn 2007). Typically, metabolic pathways are highly segregated in different subcellular organelles (Browsher and Tobin 2001; Lunn 2007). Thus, a fundamental understanding of plant metabolism requires a qualitative and quantitative description of the metabolome within different compartments of the cell. The chloroplast, cytosol and vacuole constitute the main compartments of photosynthetic and other metabolic activities, including biosynthetic and hydrolytic activities, in the leaves of plants. Knowledge of metabolic networks and their regulation can therefore be further developed based upon increased understanding of relative distributions of metabolites in these compartments as determined by experimental analyses. A cellular fractionation process, which minimizes loss or degradation of metabolites, is clearly of immense value as a prelude to such compartmentation analysis. Given the dynamic and highly labile nature of most metabolites, it is essentially that such fractionation processes are conducted under as inert an environment as possible. One of the most promising approaches to the study of compartmentalized metabolism in leaf tissue is the non-aqueous fractionation (NAQF) procedure used to separate subcellular compartments under biochemically and enzymatically inactive conditions (Gerhardt and Heldt 1984; Stitt et al. 1989). There have been many investigations in soybean leaf including for example studies on carbohydrate metabolism (Allen et al. 1998) that could, at least in principle, benefit from spatially resolved metabolic profiling. Therefore the primary objectives of this study were to (i) effectively implement NAQF for soybean leaves, (ii) profile the soybean leaf metabolome using GC-MS, and (iii) determine compartmentation at the subcellular level of the profiled metabolites, and their relative abundances in each compartment. This information will serve as an important prelude to detailed comparative experiments (unpublished studies ongoing).

\section{Materials and methods}

\subsection{Plant material}

Soybean (Glycine max Merr. cv. Kitamusume) was grown in Hokkaido University Farm, Sapporo, Japan, from May to August. Leaves were sampled from mature (10 weeks) plants early morning (from 8 to 9 a.m) and immediately plunged in liquid nitrogen to quench further metabolism. Afterwards, leaves were freeze-dried for 5 days. The major veins were removed, and samples were re-dried for two additional days for complete drying. Freeze-dried samples were stored in air-tight tubes at $-80^{\circ} \mathrm{C}$ until use. Three set of plants were used for NAQF and metabolite analysis. The NAQF was run on each set of plants to provide one biological replicate.

\subsection{Non-aqueous fractionation}

NAQF of plant material was carried out, with slight modification, by the method of Gerhardt and Heldt (1984). All operations were carried out on ice and/or $4^{\circ} \mathrm{C}$ unless otherwise stated. A sample of 300-350 mg of freeze-dried leaves was ground in a mortar and $20 \mathrm{ml}$ carbon tetrachloride $\left(\mathrm{CCl}_{4}\right)$ and $n$-heptane mixture $(d=1.28)$, dried using molecular sieve beads (4A, Merck) at 10:1 v/w ratio, was added to the sample. The mixture was sonicated for $30 \mathrm{~s} \times 3$ times with an interval of $30 \mathrm{~s}$ during which samples were cooled with ice to avoid overheating. The homogenate was filtered through a layer of quartz wool to discard coarse material and one volume of $n$-heptane was added to the filtrate, vortexed and centrifuged at $3,000 \mathrm{~g}$ for $15 \mathrm{~min}$. The supernatant was discarded and the pellet was resuspended in $3 \mathrm{ml}$ of $\mathrm{CCl}_{4}$ - $n$-heptane mixture $(d=1.28)$. Two samples of $250 \mu \mathrm{l}$ each were withdrawn for enzyme marker and metabolite analyses. The remainder was used for NAQF. An exponential gradient of $\mathrm{CCl}_{4}-n$-heptane mixtures was made by layering, from the bottom to the top, $1 \mathrm{ml}$ of the following densities: 1.59 (pure $\mathrm{CCl}_{4}$ ), 1.54, $1.49,1.44,1.40,1.36,1.32$, and 1.28. The remainder $(\sim 2.5 \mathrm{ml})$ was layered on the top of the gradient and centrifuged at 25,000 $\mathrm{g}$ for $3 \mathrm{~h}$ (Beckman Coulter, model Optima L-XP). The content of the tube was collected as six fractions, one of $\sim 1.5 \mathrm{ml}$ and five of $\sim 2 \mathrm{ml}$ each. From each fraction, $500 \mu \mathrm{l}$ was withdrawn for enzymatic analysis. To each portion one volume of $n$-heptane was added and microcentrifuged for $3 \mathrm{~min}$ at room temperature. The supernatant was discarded and the pellet was dried overnight under low pressure in a desiccator containing silica gel and paraffin. For chlorophyll content and enzyme marker activities, $500 \mu \mathrm{l}$ of $100 \mathrm{mM}$ Bicine (pH 7.8), $50 \mu \mathrm{l}$ of $5 \mathrm{mM} \mathrm{MgCl}_{2}$ and $50 \mu \mathrm{l}$ of $1 \mathrm{mM}$ EDTA were added to the sample. After a 30 s sonication pulse, samples 
were left for $5 \mathrm{~min}$ at room temperature, then microcentrifuged for $5 \mathrm{~min}$. The aqueous supernatant was collected and used for marker enzyme assays, while the pellet was used for chlorophyll determination.

\subsection{Enzymes and chlorophyll content assays}

Chlorophyll content (stroma marker) was determined by the method of Wintermans and De Mots (1965). Phosphoenolpyruvate carboxylase (PEPC) (cytosol marker) was assayed spectrophotometrically by following the rate of NADH oxidation as described by Daniel Lane et al. (1969). The assay of $\alpha$-mannosidase (vacuole marker) was determined spectrophotometrically by the method of Li (1967) based on the utilization of $\rho$-nitrophenyl- $\alpha$-D-mannoside as substrate and the liberation of $\rho$-nitrophenol.

\subsection{Extraction and derivatization for GC-MS analysis}

The six NAQF fractions were dried and the metabolites derivatized, prior to GC-MS analysis, as described by Broeckling et al. (2005) with some modifications. The dried portions of the NAQF fractions were resuspended in $500 \mu \mathrm{l}$ of water containing ribitol $\left(10 \mu \mathrm{ml}^{-1}\right)$ as internal standard, vortexed and sonicated in a sonication-bath until total resuspension of the dry pellet. The samples were then dehydrated under a low stream of nitrogen until dryness. Afterwards, the metabolites of the dry pellet were first derivatized by methoxyamination using $60 \mu \mathrm{l}$ of $15 \mathrm{mg}$ $\mathrm{ml}^{-1}$ of methoxyamine- $\mathrm{HCl}$ in pyridine (dried prior to use over molecular sieve beads), vortexed and incubated at $50^{\circ} \mathrm{C}$ for $1 \mathrm{~h}$. Subsequently, metabolites were then derivatized with $60 \mu \mathrm{l}$ of N-methyl trimethylsilylfluoroacetamide (MSTFA) $+1 \%$ trichlormethylchlorsilane (TMCS) for $1 \mathrm{~h}$ at $50^{\circ} \mathrm{C}$. The samples were then transferred to a $300 \mu \mathrm{l}$ glass insert and analyzed by GC-MS.

\subsection{GC-MS and mass spectra analyses}

Samples were analyzed by GC-MS (HP 6890 Gas chromatograph coupled with an HP 5973 MS detector and HP 6890 Series autosampler, Agilent Technologies Inc.) as described previously (Broeckling et al. 2005; Roessner et al. 2000). The mass spectral data were then analyzed using the AMDIS software (Automated Mass Spectra Deconvolution and Identification System, http://chemdata. nist.gov/mass-spectra/amdis/) and comparison was made using commercial NIST 02 Mass Spectral Library or a custom internal database generated from authentic compounds. The data files were aligned, and quantitative and qualitative data extracted using MET-IDEA software
(Noble Foundation, Ardmore. OK, USA) (Broeckling et al. 2006).

\subsection{Data and statistical analysis}

The data and calculation were obtained from the measurements of three density gradient of the same leaf material. The evaluation of the subcellular compartmentation of metabolites was performed using the deconvolution method of Riens et al. (1991) which is based upon the assumption that the metabolites are confined to the stroma, cytosol and vacuole designated by the distribution of the corresponding marker enzymes in the six fractions collected after NAQF.

Correlation analysis was performed with GraphPad Prism 4.03 software (GraphPad Software Inc., San Diego, CA, USA). Hierarchical cluster analysis (HCA) was performed with SPSS 10.0 software (SPSS Inc., Chicago, IL. USA). In order to ensure that groups share good correlation, the Pearson correlation coefficients were selected as the measurement between the three compartments, and the cluster method in use was furthest neighbor.
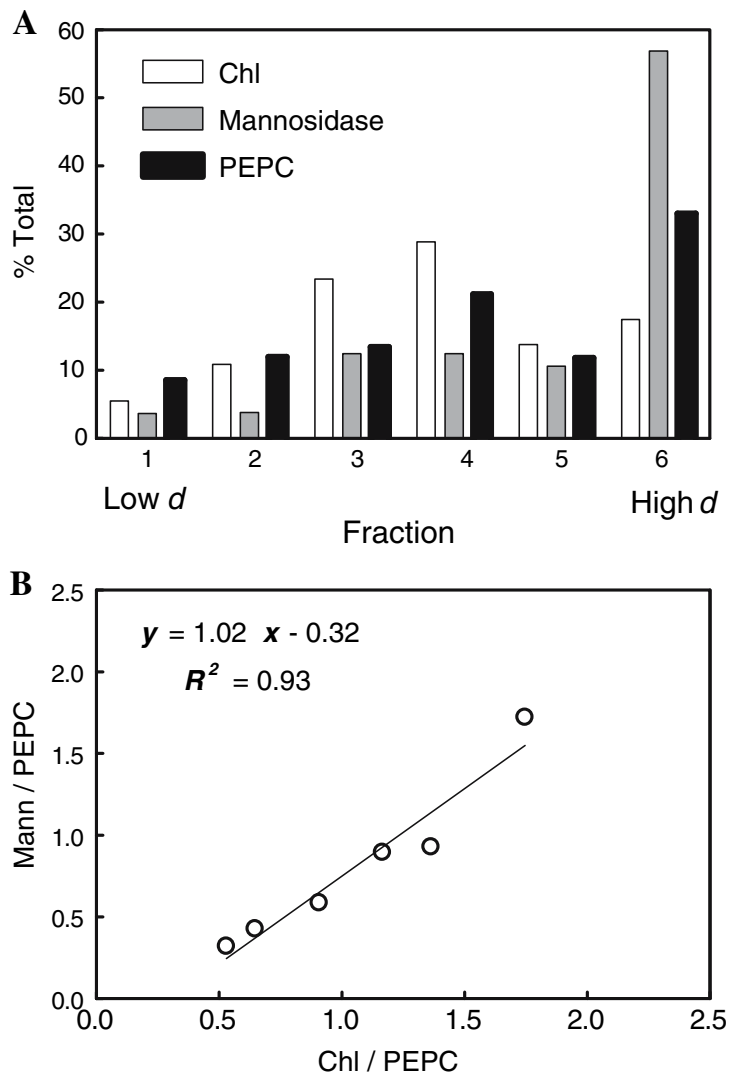

Fig. 1 Distribution and fractionation (A), and comparative regression correlations (B) of marker enzymes and chlorophylls in a non aqueous gradient obtained from dried mature soybean leaves 


\section{Results and discussion}

\subsection{Fractionation of subcellular organelles}

Subcellular fractionation, which consists of disruption of the cellular organization (homogenization) followed by fractionation of the homogenate to separate the different organelles, was applied to plant material by Gerhardt and Heldt (1984) and developed later by Stitt et al. (1989). This methodology has been consistently improved and many solvents have been recommended instead of $\mathrm{CCl}_{4}$, such as tetrachloethylene (Riens et al. 1991) or dichlorobromoethane (Hartwell et al. 2002). The former solvent was suggested because of its low toxicity, while the latter was shown to allow effective separation of vacuolar from cytosol material when applied to mature leaves of Bryophyllum.

In Fig. 1A, the chloroplastic material appeared, as expected, mainly in the middle density fractions, while the cytosolic material appeared mainly in the middle or heaviest fractions. On the other hand, the vacuolar material was concentrated in the highest density fractions, which contain more than $70 \%$ of the total activity of $\alpha$-mannosidase. The linear regression analysis of the fractionated material and collection of six fractions showed that the goodness of fit was 0.93 (Fig. 1B). The calculated $P$ value was high with a low deviation from zero intercept, indicating good reproducibility and separation into six fractions (data not shown). This good distribution is likely due to the number of fractions collected. Although previous studies made no recommendation on the number of density fractions to be collected, we suggest that six or seven fractions should be considered very appropriate for the calculation based on the model of Riens et al. (1991). Although we did not observe any significant difference in the distribution of the markers when eight fractions were collected (data not shown), however, a high $(n \geq 8)$ or low $(n \leq 5)$ number of fractions could falsify criteria for the

Table 1 Metabolites identified from non aqueous fractionated sample of soybean leaves

\begin{tabular}{|c|c|c|c|c|c|}
\hline Organic acids & Amino acids & Sugars + sugar alcohols & Fatty acids & Phenolics & Cyclitols \\
\hline 1 2-Ketoglutaric acid & 20 Arginine & 41 Arabinose & 70 Aconitic acid-trans & 81 Caffeic Acid & 90 Inositol \\
\hline 2 3-Phos ${ }^{\mathrm{p}}$ hoGlyceric acid & 21 Glutamic acid & 42 Arabitol & 71 Alantoin & 82 Calystegine & 91 Myo-Inositol \\
\hline 3 Ascorbic Acid & 22 Alanine & 43 Celobiose & $72 \mathrm{al}^{\mathrm{p}}$ ha-Linoleic Acid & 83 Coumaric Acid & 92 Phytol \\
\hline 4 Benzoic acid & 23 Asparagine & 44 D-Mannitol & 73 Butyric acid & 84 Gentistic acid & 93 Pinitol \\
\hline 5 Citric Acid & $24 \mathrm{As}^{\mathrm{p}}$ artic acid & 45 D-Mannose & 74 Decanoic Acid & 85 Quinic acid & \\
\hline 6 Dehydroascorbic acid & 25 Glutamine & 46 Erythritol & 75 Eicosanoic acid & 86 Salycilic acid & \\
\hline 7 Erythronic acid & 26 Glycine & 47 Fructose & 76 Erythronic acid & 87 Shikimic acid & \\
\hline 8 Fumaric Acid & 27 Homoserine & 48 Fructose-6-Phos ${ }^{\mathrm{p}}$ hate & 77 Linoleic acid & 88 Sinapinic acid & \\
\hline 9 Glyceric acid & 28 Isoleucine & 49 Galactose & 78 Propanoic Acid & 89 trans-Ferulic acid & \\
\hline 10 Glycolic Acid & 29 Leucine & 50 Gentiobiose & 79 Stearic Acid & & \\
\hline 11 Hexonic acid & 30 Lysine & 51 Galactitol & 80 Steric acid & & \\
\hline 12 Malic Acid & 31 Methionine & 52 Glucose & & & \\
\hline 13 Malonic Acid & 32 Norleucine & 53 Glucose-6-Phos ${ }^{\mathrm{p}}$ hate & & & \\
\hline 14 Oxalic Acid & 33 Ornitine & 54 Glucitol & & & \\
\hline 15 Phosphoric acid & 34 Phenylalanine & 55 Glycerol & & & \\
\hline $16 \mathrm{Pi}^{\mathrm{P}}$ ecolic acid & 35 Proline & 56 Gulose & & & \\
\hline 17 Ribonic acid & 36 Putrescine & 57 Maltose & & & \\
\hline 18 Succinic acid & 37 Serine & 58 Maltotriose & & & \\
\hline \multirow[t]{11}{*}{19 Threonic acid } & 38 Threonine & 59 Melezitose & & & \\
\hline & 39 Tyrosine & 60 Melibiose & & & \\
\hline & 40 Valine & 61 Rhamnose & & & \\
\hline & & 62 Ribose & & & \\
\hline & & 63 Sorbose & & & \\
\hline & & 64 Sucrose & & & \\
\hline & & 65 Tagatose & & & \\
\hline & & 66 Trehalose & & & \\
\hline & & 67 Xylitol & & & \\
\hline & & 68 Xylose & & & \\
\hline & & 69 Xylulose & & & \\
\hline
\end{tabular}


best fit for the calculation of the distribution of metabolites between the compartments. The reader is referred to Riens et al. for details of calculations used in assessing compartmental distribution of metabolites and on goodness-of-fit criteria used. Overall, the distribution of the chlorophyll and the marker enzymes in the six fractions is comparable with many other previous works carried out on leaves of spinach (Gerhardt and Heldt 1984; Riens et al. 1991; Stitt et al. 1989; Weiner et al. 1987) and pea (Sharkey and Vanderveer 1989). More specifically, Sharkey and Vanderveer (1989) suggested chlorophyll as a chloroplastic marker because they did not find significant differences between this marker and glyceraldehyde-3-P-dehydrogenase. They also noted a high proportion of chlorophyll in fractions two and three (lightest fractions) which may reflect challenges in fractionating leaf material since a typical plant cell contains fragile subcellular organelles, while being surrounded by a strong cell wall.

\subsection{Spatially profiling of metabolites by GC-MS}

To our knowledge, there are few published reports on the metabolic profiles of soybean leaves. Our GC-MS analyses of the fractionated soybean leaf material in this study identified over 200 resolved peaks. Approximately $50 \%$ of these peaks could be identified as discrete metabolites with known chemical structure (Table 1). These identified metabolites represent numerous metabolic pathways and photosynthates involved in biosynthesis and breakdown, including processes such as starch biosynthesis (e.g. hexoses and phosphorylated metabolites), starch breakdown (e.g. maltose), phenolic and related compound metabolism (e.g. shikimic acid). The identification of cyclitols (e.g. pinitol and inositol) suggests also that our plant material was exposed to environmental stress since plant leaves were harvested at maturity during a drought period during summer, although samples were collected early morning. We also noted the presence of compounds, such as ribonic acid and pipecolic acid, which are considered as intermediary moieties of specific pathways such as alkaloid biosynthesis. Thus, from a purely fundamental point of view, our metabolite profiling approach should be able to rapidly and accurately identify diverse metabolic changes and the data can contribute to understanding physiological processes that operate as a response to developmental, genetic and/or environmental changes (Lange 2006; Weckwerth and Fiehn 2002).

In addition to reporting on a large number of identified metabolites, the relative ratios of profiled metabolite classes were estimated. Approximately $30 \%$ of identified components were soluble sugars and sugar alcohols, while around 4\% were represented by inositol, myo-inositol, ascorbic acid and dehydroascorbic acid. Organic acids, primarily comprised of TCA cycle intermediates, represented $20 \%$ of the identified metabolites, fatty acids $12 \%$, amino acids $16 \%$ and other compounds (e.g. glucaric acid, galactonic acids, saccharic acid) represented 5\%, while phenolic compounds represented around $10 \%$. We also noted that the most relatively abundant metabolites in stroma, cytosol and vacuoles (see later) are: citric, succinic, malic and fumaric acids, glucose, fructose and sucrose, pinitol and myo-inositol, and, $\beta$-alanine and glycine.

\subsection{Compartmentation analysis}

The compartmentation of the major classes of metabolite was estimated by the three compartmentation analysis method of Riens et al. (1991). As illustrated by Fig. 2, sugars, organic acids and fatty acids had a greater relative abundance in the vacuole with 50, 55, and 50\%, respectively, whereas amino acids showed a greater relative abundance in the cytosol with $45 \%$. Based on the calculation method adopted here, it is apparent that the compartmental distribution showed a decreasing order from vacuole to chloroplasts: vacuole $>$ cytosol $>$ stroma. Whilst we must of course consider the interconnected issues of synthesis, transport and catabolism this decreasing order of metabolite abundance suggests, at least in the soybean leaf studied here, that photosynthates accumulate in compartments associated with biosynthetic activities i.e. the vacuole and cytosol, to a greater extent than in the chloroplasts.

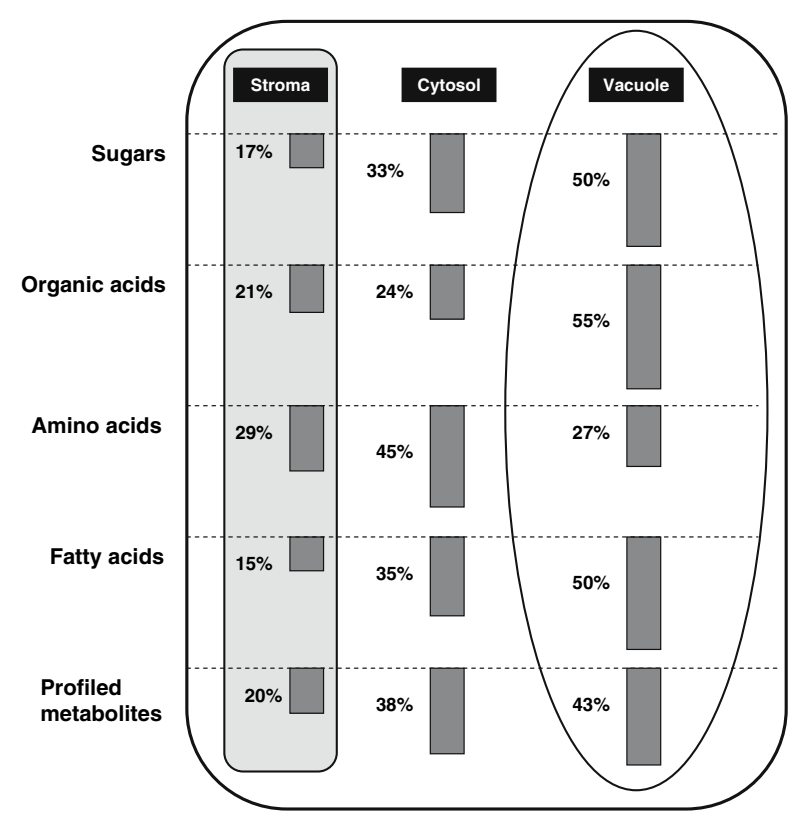

Fig. 2 Subcellular distribution of the relatively most abundant profiled metabolites in mature soybean leaves evaluated from six fraction and calculated as described in Materials and methods 
It was of considerable interest to note that not only major TCA cycle intermediates accumulate in the vacuole compartment, but other primary metabolites such as maltose and mannitol, derived from starch breakdown or physiological stress, do also. The presence of a high proportion of phenolic acids (p-coumaric, caffeic acid and ferulic acid) in the vacuole also implies consistent phenylpropanoid biosynthesis suggesting metabolism induced by one or more biotic or abiotic stresses (Dixon and Pavla 1995; Wingler et al. 2000). The phenylpropanoid pathway leads to lignin biosynthesis suggesting, at least in part, the beginning of senescence. The low levels of glutamic acid and alanine in the vacuolar compartment (Table 2) and the high levels of glycine in chloroplast compartment (Table 2) may suggest low storage of nitrogen in leaves, and the ready export of amino acid metabolites, as it has been earlier reported that amino acids accumulation is greater in the sink than in the source in soybean leaves (VerNooy and Lin 1986).

These and other findings highlight the complexity of metabolism where all pathways are interconnected and plant tissues are comprised of more than one cell type giving heterogenic organelle preparations (Lunn 2007). Only through greater spatial resolution can we understand the role of subcellular synthesis and different transport phenomena in plants. As such, this highlights the value in studying metabolome compartmentation. This can be illustrated by considering sucrose which, as we all know, has a central role in carbohydrate metabolism. A vast literature reports that sucrose metabolism takes place in the cytosol (Baroja-Fernández et al. 2001; Koch 1996). Its distribution in the cytosol was shown to be $100 \%$ in
Table 2 Subcellular distribution of the relatively most abundant and important metabolites profiled in mature soybean leaves. Estimation was calculated using the threecompartment calculation as described in Sect. 2

\begin{tabular}{|c|c|c|c|c|}
\hline & & Stroma & Cytosol & Vacuole \\
\hline \multirow[t]{13}{*}{ Cluster A } & 3 Succinic acid & 27 & 38 & 35 \\
\hline & 28 Pinitol & 27 & 39 & 34 \\
\hline & 11 Propionic acid & 26 & 40 & 34 \\
\hline & 29 Myo-inositol & 32 & 38 & 31 \\
\hline & 13 Glucose & 29 & 31 & 40 \\
\hline & 9 Glutamic acid & 21 & 50 & 29 \\
\hline & 19 Ribose & 26 & 49 & 25 \\
\hline & 12 Stearic acid & 18 & 57 & 24 \\
\hline & 15 Sucrose & 25 & 55 & 21 \\
\hline & 8 Alanine & 30 & 61 & 9 \\
\hline & 21 Xylulose & 36 & 48 & 16 \\
\hline & 1 Oxalic acid & 52 & 29 & 18 \\
\hline & 7 Glycine & 44 & 29 & 27 \\
\hline \multirow[t]{11}{*}{ Cluster B } & 14 Fructose & 12 & 27 & 61 \\
\hline & 23 Xylitol & 11 & 26 & 64 \\
\hline & 5 Malic acid & 12 & 24 & 62 \\
\hline & 4 Fumaric acid & 11 & 23 & 66 \\
\hline & $25 \alpha$ ketoglutaric acid & 10 & 32 & 58 \\
\hline & 26 Ascorbic acid & 12 & 30 & 59 \\
\hline & 16 Maltose & 7 & 35 & 58 \\
\hline & 17 Galactose & 24 & 27 & 49 \\
\hline & 22 Arabinose & 22 & 31 & 47 \\
\hline & 10 Butyric acid & 14 & 36 & 50 \\
\hline & 20 Cellobiose & 12 & 42 & 46 \\
\hline \multirow[t]{8}{*}{ Cluster C } & 6 Citric acid & 5 & 17 & 78 \\
\hline & 18 Mannose & 7 & 17 & 76 \\
\hline & 31 Ferulic acid & nd & 22 & 78 \\
\hline & 24 Mannitol & nd & nd & 100 \\
\hline & 27 Dehydroascorbic acid & nd & nd & 100 \\
\hline & 2 Malonic acid & nd & 8 & 92 \\
\hline & 32 Caffeic acid & nd & 9 & 91 \\
\hline & $30 \rho$-Coumaric acid & nd & 12 & 88 \\
\hline
\end{tabular}


spinach leaves (Riens et al. 1991), while in potato tubers the figure is only 17\% (Farré et al. 2001). New findings in the regulation of carbohydrate continue apace. For example, it was recently hypothesized that the presence of maltose during light periods is a bridge between transitory starch breakdown and the plant's adaptation to change in environmental conditions. Since the biochemical pathways of synthesis and degradation of transitory starch is still not fully understood ( $\mathrm{Lu}$ and Sharkey 2006; Zeeman et al. 2004), a role for spatial metabolic profiling methods is clearly suggested.

Hierarchical cluster analysis was also performed on the highest abundant metabolites from the total identified metabolites (Fig. 3). The distribution of these metabolites is shown in Table 2. We noted that first cluster (A) contains compounds which are present at higher relative abundances in the cytosol, while cluster B and cluster C comprise the most abundant compounds in the vacuole and stroma, respectively. Surprisingly and given the variability of the different metabolites, we did not observe from one compartment any value for a given metabolite less than 5\% of the overall abundance. Values $\leq 20 \%$ represented only $17 \%$ of the total values reported in Table 2. Given this variability within the calculated values, we might suggest that it is not appropriate to consider a distribution value below $5 \%$ and this value could constitute the limit of detection required to presume the presence of a given metabolite.

Whilst the compounds in the cluster A are relatively abundant in the cytosol, their relative distribution in stroma and vacuole still represents over $20 \%$ of total abundance. Cluster A also included stress related compounds such as pinitol and myo-inositol. We noted that a great portion of glutamic acid and alanine was compartmentalized in the cytosol, while a greater portion of glycine was observed in the stroma (Cluster $\mathrm{C}$ ). The greater portion of glutamic acid and alanine in the cytosol is likely due to the difficult situation where no appropriate separation of the mitochondria and the cytosol compartments has been achieved using NAQF procedure (Gerhardt and Heldt 1984). The reactions of amino acids are compartmentalized mainly in mitochondria (Oaks and Bidwell 1970) although there is evidence that many enzymes involved in amino acid biosynthesis are found also in the cytosol (Miflin and Lea 1977).

The compounds in cluster B are abundant and preferentially compartmentalized in the vacuole, while their relative abundances in the stroma are very low except for glucose. Cluster B included the main part of sugars and almost total secondary metabolites indicating that major carbohydrate, flavonoid and phenylpropanoid accumulation occurs in the vacuole during this mature stage. The
Fig. 3 Dendrogram showing the Hierarchical Cluster Analysis (HCA) of the relatively most abundant and important profiled metabolites in mature soybean leaves. HCA was done on extracts obtained from non-aqueous fractionated plant material and subcellular distributed metabolites

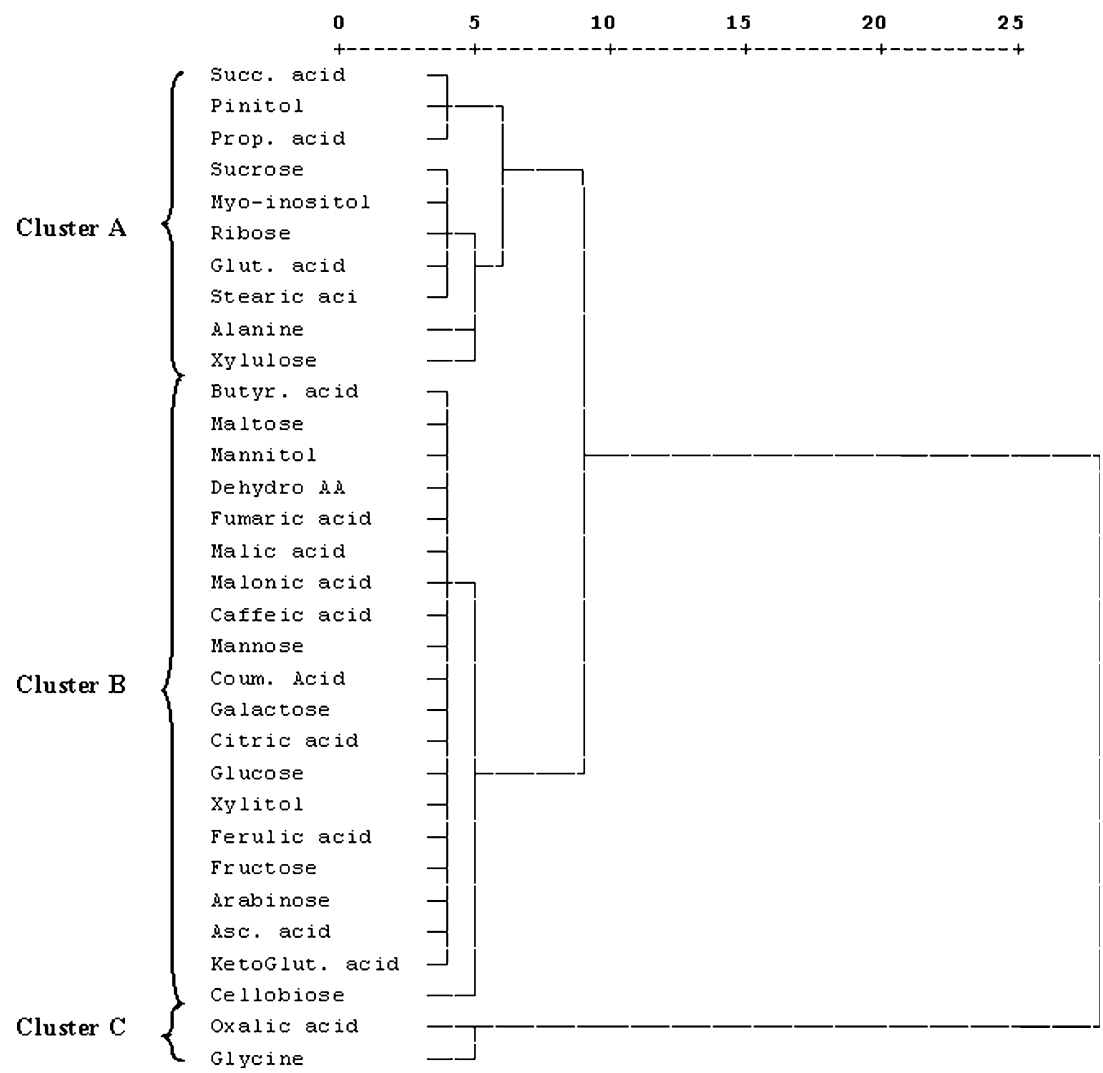


greatest levels of TCA cycle organic acids were found to be in vacuole, and this suggests that there is a large pool of organic acids located in this organelle, while a relatively small one in other organelles. This high amount of organic acids in vacuole has also been reported in many species (Martinoia and Rentsch 1994; Martinoia et al. 2000). As for organic acids, major sugars also accumulate in vacuole, because it is generally admitted that these organelles are storage compartments of a large variety of organic compounds, and have multiple functions (Martinoia et al. 2007; Matile 1977).

Cluster $\mathrm{C}$ is minor and the two compounds are distributed one half in stroma and one half is almost equally divided between cytosol and vacuole. Biologically, no specific metabolic pathway, such as glycolate pathway, suggests itself. We did not confirm the presence of glyoxylate, easily oxidized to oxalic acid (Richardson and Tolbert 1961), although glycolate is known to play a role as an activator of fatty acid biosynthesis (Castelfranco et al. 1955).

\section{Concluding remarks}

In conclusion, a procedure for NAQF and GC-MS analysis of soybean leaf tissue as described above has been described. A large number of metabolites have been identified and our preliminary analysis suggests we can demonstrate the degree of subcellular compartmentalization of these metabolites. This can, at least in principle, lead to hypotheses on the role of compartmentalization in the regulation of metabolite synthesis. Comparative analyses of plants exposed to different abiotic and biotic stresses will prove particularly useful and unpublished studies are pending.

Acknowledgments The authors would like to thank Dr L.W. Sumner, and The Samuel Roberts Noble Foundation, Ardmore, Oklahoma, USA, for their hosting and their technical assistance to Dr. N. Benkeblia. The assistance of Dr. E. Urbanczyk-Wochniak is also acknowledged. This research was partially supported by the Ministry of Education, Science, Sports and Culture, Grant-in-Aid for Scientific Research (B), 17658144, 2005.

\section{References}

Allen, L. H., Bisbal, E. C., \& Boote, K. J. (1998). Nonstructural carbohydrates of soybean plants grown in subambiant and superambiant levels of $\mathrm{CO}_{2}$. Photosynthesis Research, 56, 143-155.

Baroja-Fernández, E., José Muñoz, F., Akazawa, T., \& PozuetaRomero, J. (2001). Reappraisal of the currently prevailing model of starch biosynthesis in photosynthetic tissues: A proposal involving the cytosolic production of ADP-glucose by synthase and occurrence of cyclic turnover of starch in chloroplast. Plant Cell, 42, 1311-1319.

Broeckling, C. D., Huhman, D. V., Farag, M. A., Smith, J. T., May, G. D., Mendes, P., Dixon, R. A., \& Sumner, L. W. (2005). Metabolic profiling of Medicago truncatula cell cultures reveals the effects of biotic and abiotic elicitors on metabolism. Journal of Experimental Botany, 56, 323-336.

Broeckling, C. D., Reddy, I. R., Duran, A. L., Zhao, X., \& Sumner, L. W. (2006). MET-IDEA: a data extraction tool for mass spectrometry-based metabolomics. Analytical Chemistry, 78, 4334-4341.

Brown, M., Dunn, W. B., Ellis, D. I., Goodacre, R., Handl, J., Knowles, J. D., O’Hagan, S., Spasić, I., \& Kell, D. B. (2005). A metabolome pipeline: From concept to data to knowledge. Metabolomics, 1, 39-51.

Browsher, C. G., \& Tobin, A. K. (2001). Compartmentation of metabolism within mitochondria and plastids. Journal of Experimental Botany, 52, 513-527.

Castelfranco, P., Stumpf, P. K., \& Contopoulou, R. (1955). Fat metabolism in higher plants. The Journal of Biological Chemistry, 217, 567-277.

Daniel Lane, M., Maruyama, H., \& Easterday R. L. (1969). Phosphoenolpyruvate carboxylase from peanut cotyledons. Methods in Enzymology, 13, 277-283.

Dixon, R. A., \& Pavla, N. L. (1995). Stress-induced phenylpropanoid metabolism. Plant Cell, 7, 1085-1097.

Dunn, W. B., \& Ellis, D. I. (2005). Metabolomics, current analytical platforms and methodologies. Trends in Analytical Chemistry, 24, 285-294.

Farré, E. M., Tiessen, A., Roessner, U., Geigenberg, P., Trethewey, R. N., \& Willmitzer, L. (2001). Analysis of the compartmentation of glycolytic intermediates, nucleotides, sugars, organic acids, amino acids, and sugat alcohols in potato tubers using a nonaqueous fractionation method. Plant Physiology, 127, $685-700$.

Fiehn, O., Kopka, J., Dörmann, P., Altmann, T., Trethewey, R. N., \& Willmitzer, L. (2000). Metabolite profiling for plant functional genomics. Nature Biotechnol, 18, 1157-1161.

Fridman, E., \& Pichersky, E. (2005). Metabolomics, genomics, proteomics, and the identification of enzymes and their substrates and products. Current Opinion in Plant Biology, 8, 242-248.

Gerhardt, R., \& Heldt, H. W. (1984). Measurement of subcellular metabolite levels in leaves by fractionation of freeze-stopped material in nonaqueous media. Plant Physiology, 75, 542-547.

Goodacre, R., Vaidyanathan, S., Dunn, W. B., Harrigan, G. G., \& Kell, D. B. (2004). Metabolimics by numbers: acquiring and understanding global metabolite data. Trends in Biotechnology, $22,245-252$.

Hartwell, J., Nimmo, G. A., Wilkins, M. B., Jenkins, G. I., \& Nimmo, H. G. (2002). Probing the circadian control of phosphoenolpyruvate carboxylase kinase expression in Kalanchö̈ fedtschenkoi. Functional Plant Biology, 29, 663-668.

Kell, D. (2004). Metabolomics and systems biology: Making sense to the soup. Current Opinion in Microbiology, 7, 296-307.

Koch, K. E. (1996). Carbohydrate-modulated gene expression in plants. Annual Review of Plant Physiology and Plant Molecular Biology, 47, 509-540.

Lange, B. M. (2006). Integrative analysis of metabolic networks: From peaks to flux model. Current Opinion in Plant Biology, 9, 220-226.

Li, Y. (1967). Studies on the glycosidases in Jack bean meal. The Journal of Biological Chemistry, 26, 5474-5480.

Lu, Y., \& Sharkey, T. D. (2006). The importance of maltose in transitory starch breakdown. Plant, Cell and Environment, 29, 353-366. 
Lunn, J. E. (2007). Compartmentation in plant metabolism. Journal of Experimental Botany, 58, 543-547.

Martinoia, E., \& Rentsch, D. (1994). Malate compartmentationresponses to a complex metabolism. Annual Review of Plant Physiology, 45, 447-467.

Martinoia, E., Massonneau, A., \& Frangne, N. (2000). Transport processes of solutes across the vacuolar membrane of higher plants. Plant Cell Physiology, 41, 1175-1186.

Martinoia, E., Maeshima, M., \& Neuheus, E. (2007). Vacuolar transporters and their essential role in plant metabolism. Journal of Experimental Botany, 58, 83-102.

Matile, P. (1977). Biochemistry and function of vacuoles. Annual Review of Plant Physiology, 29, 193-213.

Miflin, B. J., \& Lea, P. J. (1977). Amino acid metabolism. Annual Review of Plant Physiology, 28, 299-329.

Oaks, A., \& Bidwell, R. G. S. (1970). Compartmentation of intermediary metabolites. Annual Review of Plant Physiology, 21, 43-66.

Richardson, K. E., \& Tolbert, N. E. (1961). Oxidation of glyoxilic acid to oxalic acid by glycolic acid oxidase. The Journal of Biological Chemistry, 236, 1280-1284.

Riens, B., Lohaus, G., Heineke, D., \& Heldt, H. W. (1991). Amino acid, and sucrose content determination in the cytosolic, chloroplastic, and vacuolar compartments and in the phloem sap of spinach leaves. Plant Physiology, 97, 227-233.

Roessner, U., Wagner, C., Kopka, J., Trethewey, R. N., \& Willmitzer, L. (2000). Simultaneous analysis of metabolites in potato tuber by gas chromatography-mass spectrometry. The Plant Journal, $23,131-142$.

Sharkey, T. D., \& Vanderveer, P. J. (1989). Stromal phosphate concentration is low during feedback limited photosynthesis. Plant Physiology, 91, 679-684.

Stitt, M., Lilley, R. McC., Gerhardt, R., \& Heldt, H. (1989). Metabolite level in specific cells and subcellular compartments of plant leaves. Methods Enzymology, 174, 518-552.

VerNooy, C. D., \& Lin, W. (1986). Amino acid transport in protoplast isolated from soybean leaves. Plant Physiology, 81, 8-11.

Weckwerth, W., \& Fiehn, O. (2002). Can we discover novel pathways using metabolomic analysis? Current Opinion in Biotechnology, 13, 156-160.

Weiner, H., Stitt, M., \& Heldt, H. W. (1987). Subcellular compartmentation of pyrophosphate and alkaline pyrophosphate in leaves. Biochimica et Biophysica Acta, 893, 13-21.

Wingler, A., Lea, P. J., Quick, W. P., \& Leegood, R. C. (2000). Photorespiration: metabolic pathways and their role in stress protection. Philosophical Transactions of the Royal Society of London. Series B, 355, 1517-1529.

Wintermans, J. F. G. M., \& De Mots, A. (1965). Spectrophotometric characteristics of chlorophylls $a$ and $b$ and their pheophytins in ethanol. Biochimica et Biophysica Acta, 109, 448-453.

Zeeman, S. C., Smith, S. M., Smith, A. M. (2004). The breakdown of starch in leaves. The New Phytologist, 163, 247-261. 